Cinémas

Revue d'études cinématographiques

Journal of Film Studies

\title{
Le rôle de la musique dans la définition du cinéma comme art : à propos de l'avant-garde des années 20
}

\section{Dominique Château}

Volume 3, numéro 1, automne 1992

Cinéma et Musicalité

URI : https://id.erudit.org/iderudit/1001181ar

DOI : https://doi.org/10.7202/1001181ar

Aller au sommaire du numéro

Éditeur(s)

Cinémas

ISSN

1181-6945 (imprimé)

1705-6500 (numérique)

Découvrir la revue

Citer cet article

Château, D. (1992). Le rôle de la musique dans la définition du cinéma comme art : à propos de l'avant-garde des années 20. Cinémas, 3(1), 78-94.

https://doi.org/10.7202/1001181ar
Résumé de l'article

L'un des thèmes récurrents du discours de l'avant-garde cinématographique des années 20 est la recherche d'une forme de cinéma qui le classe dans la catégorie de l'art. Si la peinture sert principalement de modèle dans cette recherche d'un cinéma artistique, la musique intervient également. Cette intervention revêt plusieurs aspects que l'auteur tente ici d'analyser, en montrant qu'ils constituent différentes sortes de modèle pour le cinéma artistique. L'auteur s'interroge aussi sur le fait que le recours au modèle musical, en relation avec le modèle pictural, s'intègre, en apparence paradoxalement, à la recherche de la pureté du langage cinématographique, de son essence. Deux axes de pensée se combinent alors : la question de l'art et la question du médium. 


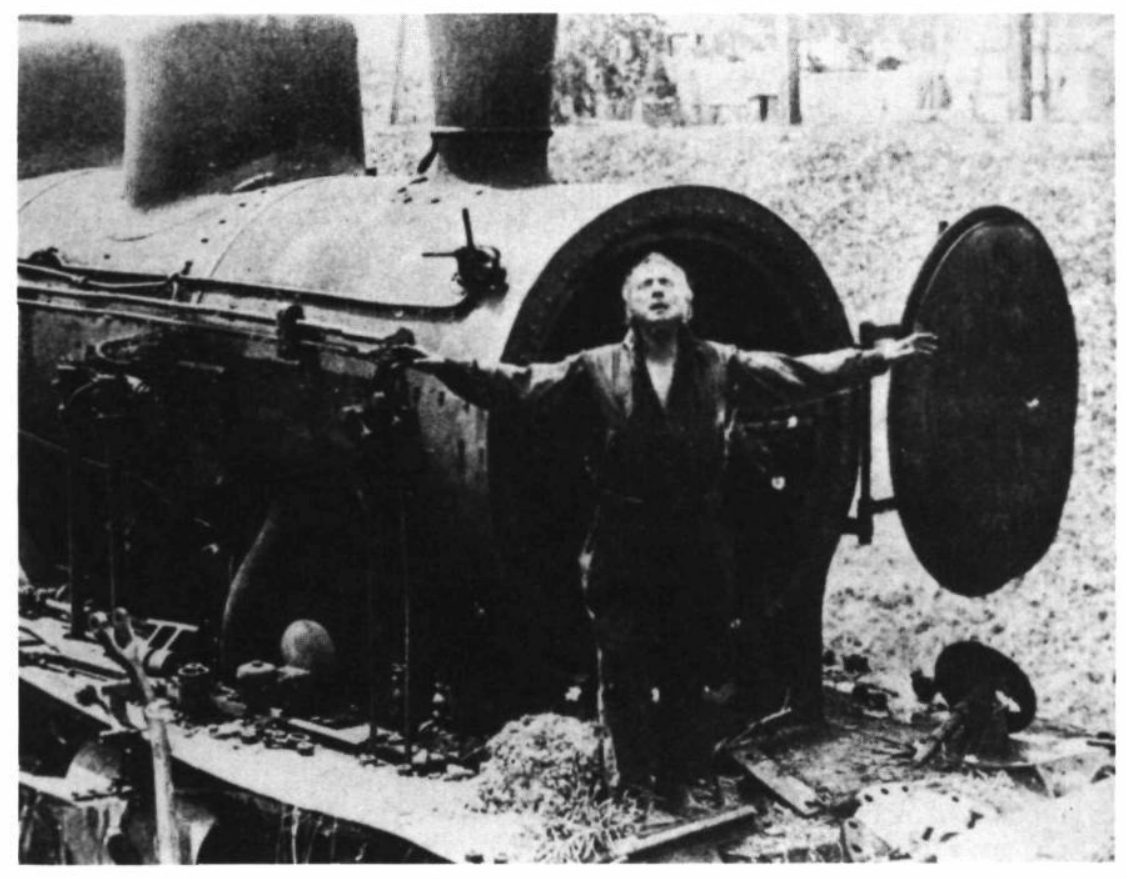

La Roue d'Abel Gance (1923)

Coll. Cinémathèque québécoise 


\title{
Le rôle de la musique dans la définition du cinéma comme art : à propos de l'avant-garde des années 20
}

\section{Dominique Château}

\begin{abstract}
RÉSUMÉ
L'un des thèmes récurrents du discours de l'avant-garde cinématographique des années 20 est la recherche d'une forme de cinéma qui le classe dans la catégorie de l'art. Si la peinture sert principalement de modèle dans cette recherche d'un cinéma artistique, la musique intervient également. Cette intervention revêt plusieurs aspects que l'auteur tente ici d'analyser, en montrant qu'ils constituent différentes sortes de modèle pour le cinéma artistique. L'auteur s'interroge aussi sur le fait que le recours au modèle musical, en relation avec le modèle pictural, s'intègre, en apparence paradoxalement, à la recherche de la pureté du langage cinématographique, de son essence. Deux axes de pensée se combinent alors : la question de l'art et la question du médium.
\end{abstract}

\begin{abstract}
One of the recurrent themes in the discourse of the avantgarde cinematography of the $1920 \mathrm{~s}$ is the search for a form of cinema that will merit classification as art. Though painting is the primary model in the search for an artistic cinema, music also plays a role. This role has several dimensions, which the author analyses here in a discussion of the different kinds of model they constitute for artistic cinema. The author also raises the question of how, despite the apparent paradox, this recourse to a musical model, in conjunction with the pictural model, can be integrated with the search for purity of cinematographic language, for its essence. Two lines of analysis are here pursued : the question of art and the question of the medium.
\end{abstract}


«Le cinéma parlant ne tiendra pas, (...) ce sera surtout une question de mode» (de Hass, p. 268), prophétisait Henri Chomette en 1929. En fait de mode, aux alentours de 1930, un médium change de définition : non seulement le cinéma, ce «muet de naissance», comme disait Tristan Bernard, accède à la parole, mais le parlant prend la place, toute la place, du muet, un peu comme si l'opéra, à sa naissance, s'était intégralement substitué à la musique. Il faut se rendre à l'évidence : l'unité de l'histoire du cinéma se fonde sur la puissance de l'industrie, sur la logique du profit, en vertu de quoi les modèles de comportement artistique qui tentaient de s'exprimer avant la date fatidique se heurtent désormais à des obstacles de plus en plus insurmontables. À l'exemple d'un René Clair, chez qui le désir de faire des films l'emporte progressivement sur la fidélité à l'héritage avant-gardiste, le cinéma se détache de l'environnement artistique pour s'isoler dans son propre monde.

Or, ce gain d'autonomie est interprété par les avant-gardistes comme une perte d'intégrité, ce qui peut surprendre au regard de ce que nous apprend l'histoire des autres arts. Pour la peinture, par exemple, la conquête de l'autonomie (avec, à son terme, l'art abstrait) semble plutôt coïncider avec la concentration de ce médium sur son domaine propre. Cependant, ceux pour qui l'indépendance du monde du cinéma est corrélative à la corruption du langage cinématographique, ne semblent pas manifester une conception très claire de l'intégrité. D'une part, ils luttent ou, du moins, prétendent lutter contre toute influence extrinsèque, en fait essentiellement littéraire ou théâtrale; d'autre part, nombre d'entre eux préconisent de penser le cinéma dans les termes de la peinture ou de la musique. Il y a, d'un côté, des arts dont le modèle serait néfaste, de l'autre, des arts dont le modèle serait profitable; d'un côté, des arts antinomiques avec le cinéma, de l'autre, des arts analogues ou proches de lui. On comprendrait aisément la répartition si elle correspondait à une classification des médias selon le critère de l'ordre sensoriel : à cet égard, le muet va avec la peinture, mais comment peut-on l'apparier avec la musique?

Non seulement la recherche de pureté ne s'embarrasse pas de cette difficulté, mais encore elle cultive le paradoxe. La musique offrirait le meilleur modèle d'un cinéma débarrassé de toute influence extrinsèque, d'un cinéma pur. Ici, le modèle a le sens d'une analogie conceptuelle, suivant laquelle transposer l'idéal de la pureté musicale au cinéma, c'est réaliser cet idéal avec les moyens propres de ce médium. Mais le mot peut avoir un autre sens : celui d'un système de règles susceptible d'être appliqué dans un autre domaine que son domaine d'origine; dans ce cas, le cinéma atteindrait l'idéal de pureté en utilisant les modes de la composition 
musicale. La pensée de l'avant-garde des années 20 flotte entre ces deux interprétations, entre l'imitation de la pureté de la musique et l'imitation de ses procédés. En tout état de cause, ces deux opinions se rejoignent dans une commune condamnation de l'adjonction de la musique au film. Sa musicalité, le film muet est censé la receler en lui-même. On va même jusqu'à dire que le film est en soi plus musical que la musique d'accompagnement dont on l'affuble...

Parvenu à ce degré de dissonance conceptuelle, on peut être tenté d'abandonner l'avant-garde à ses contradictions qui paraissent, d'ailleurs, expliquer son échec. Il y a, au contraire, des raisons de les considérer et de chercher à les comprendre, ainsi que je vais m'efforcer de le montrer dans cet article. Notamment, il appert que l'intromission de la musique dans le débat sur la pureté cinématographique est une contribution importante à la question du cinéma comme art et, au-delà, à l'élucidation du concept d'art en général. C'est pourquoi mon propos ne visera pas tant à faire l'inventaire exhaustif des discussions de l'époque qu'à les replacer dans le cadre de l'héritage conceptuel d'où elles procèdent peu ou prou consciemment ${ }^{1}$.

Apollinaire, dans son panégyrique du cubisme, avance en 1913 l'idée de l'autonomie de la peinture en tant que concentration sur les qualités spécifiques du tableau : «Les peintres nouveaux, écritil, procureront à leurs admirateurs des sensations artistiques uniquement dues à l'harmonie des lumières impaires» (p.59). De manière comparable, Germaine Dulac avance l'idée qu'«un vrai film ne doit pas se raconter puisqu'il doit puiser son principe actif et émotif dans des images faites d'uniques vibrations visuelles» ( $p$. 34). Or, le poète ne craint pas d'introduire dans sa caractérisation de la peinture pure le modèle de la musique :

On s'achemine ainsi vers un art entièrement nouveau, qui sera à la peinture, telle qu'on l'avait envisagéc, ce que la musique est à la littérature. Ce sera de la peinture pure, de même que la musique est de la littérature pure (Apollinaire, p. 59).

On observe dans ce raisonnement analogique, corrélativement à l'exaltation du modèle musical, la dévaluation de la littérature, ce qui ne laisserait pas de surprendre de la part d'un poète, n'était la différenciation entre poésie et littérature qu'elle présuppose. Sousjacente, en effet, il y a la conception d'une poésie pure inaugurée par Mallarmé qui ne craignait pas, lui-même, de convoquer l'idée d'une affinité entre elle et la musique comme modèle de la pureté : 
(...) oublions la vieille distinction, entre la Musique et les Lettres

(...): l'une évocatoire de prestiges situés à ce point de l'ouïe et presque de la vision abstraite, devient l'entendement; qui, spacieux, accorde au feuillet d'imprimerie une portée égale (p. 359).

Il faut dire que la musique bénéficie d'un privilège depuis l'antiquité, y compris chez Platon, en dépit de sa méfiance envers ce que l'on appellera ultérieurement les beaux-arts. La musique semble, d'emblée, l'art pur par excellence, en raison de sa relation et même de son appartenance (longtemps affirmée à la suite des pythagoriciens) aux mathématiques, laquelle fonde son émancipation vis-à-vis de la mimesis. Ainsi, pour Apollinaire, ce que l'on entend dans un concert, ce ne sont pas des bruits de la nature, mais des sons artificiels qui produisent un plaisir spécifique. Le modèle de la musique a deux ressorts: négativement, il réfute la mimesis, positivement, il exemplifie l'abstraction.

Pour Kandinsky, «chaque art en arrive peu à peu au point où, grâce aux moyens qui lui appartiennent en propre, il devient capable d'exprimer ce qu'il est seul qualifié pour dire» (p. 75). À l'époque où il écrit, selon lui, l'art en général se détourne du naturalisme, de l'imitation des modèles extérieurs pour viser l'abstraction, le modèle intérieur. Les différents arts se comparent à l'aune de cette évolution et, parmi eux, la musique joue censément le premier rôle :

Depuis des siècles, la musique est par excellence l'art qui exprime la vie spirituelle de l'artiste. Ses moyens ne lui servent jamais, en dehors de quelques cas exceptionnels où elle s'est écartée de son véritable esprit, à reproduire la nature, mais à donner une vie propre aux sons musicaux (pp. 75-76).

Il est normal que le peintre, qui vise la même finalité :

(...)se tourne vers cet art et qu'il s'elforec, dans le sien, de découvrir des procédés similaires. De là, en peinture, l'actuelle recherche du rythme, de la construction abstraite, mathématique et aussi la valeur qu'on attribue aujourd'hui à la répétition des tons colorés, au dynamisme de la couleur (p. 76).

S'il ne s'agit pas de transplanter en peinture les procédés de la musique, mais d'utiliser leur modèle pour développer la spécificité des autres arts, il est clair que la possibilité de cette forme d'imitation entre les arts repose sur la présence de traits généraux qui sont comme un dénominateur commun ${ }^{2}$. Or, ces traits - le rythme, le dynamisme - la musique les comporte physiquement, sans considération du récepteur. Il faut un spectateur pour que la peinture les intègre. L'abstraction, au sens de Kandinsky, repose 
sur un paradoxe autour duquel son œuvre travaillera sans cesse, oscillant entre deux conceptions de l'art abstrait : le géométrique et le lyrique, le statique et le dynamisme.

Le cinéma, cette «peinture animée», comporte physiquement les traits de la musique qui s'ajoutent aux traits de la peinture. Certains pensent même qu'il ne se contente pas d'intégrer ces deux arts, mais réunit tous les arts, auquel cas le rapport avec la musique ne serait qu'un aspect d'un phénomène plus global. Canudo appelle septième art le cinéma, par qu'il «représente la puissance synthèse de tous les Arts : arts plastiques en mouvement rythmique, arts rythmiques en tableaux et en sculptures de lumières» (Lherminier, p. 29) ${ }^{3}$. On voit poindre, sans surprise, la notion de rythme comme noyau de la rencontre entre cinéma et musique, puisque ce paramètre préexiste dans les arts dont le septième est censé faire l'amalgame. L'histoire du cinéma serait donc l'aboutissement de l'histoire de chacun des arts qu'il intègre et absorbe : «Nous avons besoin de cinéma pour créer l'art total vers lequel tous les autres, depuis toujours, ont tendu» (Canudo, 1923a: p. 2). On notera, à ce propos, que, vue du point de vue de l'avant-garde, l'histoire du cinéma s'intègre à une large vision esthétique, tandis que, vue du point de vue du «cinéma dominant», elle se renferme sur son monde propre. Mais cette prétention totalisante du cinéma comme «carrefour des arts» (de Haas, p. 123) selon l'expression d'Abel Gance $^{4}$, peut se retourner contre le septième art, si on l'interprète comme un éclectisme : Léon Pierre-Quint (pp. 6-8) lui reproche d'emprunter «au roman une intrigue, au théâtre ses acteurs, à la musique l'accompagnement et le rythme, à la peinture ses décors, aux journaux amusant leurs sous-titres», donc de n'être qu'un plagiaire toujours inférieur à ses modèles, un art entretenu (au sens où on le dit d'une femme). D'autre auteurs tirent du même constat un impératif pour le développement de cet art étiqueté septième : non seulement prendre sa place dans la classification légitimante des arts, mais surtout y trouver le rang qui lui est propre. En 1925, Germaine Dulac se plaint que le cinéma ne soit :

(...) que le reflet des autres arts. Or, il est trop grand pour ne rester qu'un reflet, il faut le libérer de ses chaînes, et lui donner sa réelle personnalité. Dans sa technique, rien ne l'apparente aux autres arts existants. (...) Il faut nous aider à libérer le cinéma de ses entraves et créer le cinéma pur (de Haas, p. 124).

En 1927, elle réitère la plainte, constatant que le septième art n'est:

(...) qu'un succédané, une image animée mais seulement une image des expressions évoquées par la littérature, la musique, la danse, la sculpture. Il n'est pas un art. Or, dans son essence même, il en est un très grand» (1927a: p. 30). 
Ces deux citations, qui expriment une idée susceptible d'être à nouveau réitérée, puisque l'existence parallèle du cinéma narratifcommercial exhibe sans discontinuer un repoussoir, montrent que la question de l'art et la question de la pureté sont étroitement liées dans l'esprit de l'auteur comme elles le sont pour nombre de ses congénères. La condition nécessaire pour qu'un moyen d'expression reçoive le qualificatif d'art, c'est qu'il parvienne à un état d'autonomie qui lui permet de développer ses propriétés spécifiques: «Tout art porte en soi une personnalité, une individualité d'expression qui lui confère sa valeur et son indépendance» écrit encore Dulac (p. 29).

En fait, les positions des «éclectiques» et celle des «autonomistes» se rejoignent en ceci que, synthèse ou indépendance, le processus d'accession du cinéma au statut d'art en fait une forme d'art supérieure aux autres, notamment en vertu de sa définition technologique : la caméra est un «cerveau en métal, (...) un artiste» dit Jean Epstein (p. 38), un «perfectionnement du cerveau humain» renchérit Emile Vuillermoz (1927, n.p.). Pour cette même raison, il ne craint rien du théâtre, art de synthèse qui pourrait sembler rivaliser avec lui : «Le théâtre est au cinéma, écrit Picabia, ce qu'est la chandelle à la lampe électrique, l'âne à l'automobile, le cerf-volant à l'aréoplane» (p. 4). Le fait que le cinéma se fonde sur un mécanisme de reproduction induit une idée comparable à celle des théoriciens de la Renaissance, tel Léonard de Vinci, pour lesquels, en possession de la perspective, la peinture est une science de la nature capable de rivaliser avec elle : le cinéma, pour Canudo,

(...) est né de la volonté, et de la science et de l'art des hommes modernes pour exprimer plus intensément la vie, pour signifier, à travers les espaces et les temps, le sens de la vie perpétuellement neuve» (1921b : n.p.).

Cependant, l'identification du cinéma à l'appareil récepteur de la réalité, à l'œil, est dangereuse; elle peut fonder le plus plat des réalismes. Aux admirateurs de la caméra-œil ou de la caméracerveau répondent ceux qui considèrent que l'œil-caméra ne se réduit pas à la "vision toute mécanique d'objets morts» (Ramain, 1926 : p. 8), qu'il a une «vision propre, grâce à la lumière et au mouvement» (Fescourt-Bouquet, p. 19); c'est à la fois un «œil de mouche» qui voit mieux le visible (Vuillermoz, $1917:$ p. 3) et un «œil surréel» qui peut voir l'invisible (Gorelof, p. 23) ${ }^{5}$. Encore faut-il, comme le souligne Paul Ramain, que derrière l'œil inerte de la caméra, il y ait «l'œil vraiment vivant d'un artiste vibrant qui communique sa volonté (...)»(p. 8). 
En effet, la lumière ou le mouvement enregistrés peuvent n'être, comme dans les films Lumière, qu'une trace d'un spectacle réel, en sorte que l'objectif d'un cinéma d'art qui vise au-delà de ce naturalisme ne dépend pas de la seule définition du médium. Il dépend de choix effectués vis-à-vis de ses possibilités : l'avantgarde privilégie le gros plan, le plan court, le ralenti et l'accéléré, la surimpression, etc., soit une gamme de procédés qui manifestent la volonté, plutôt que de restituer le réel, de le maîtriser suivant un projet préalable. Lorsque ce projet s'identifie à un scénario, le cinéma s'écarterait de sa vocation propre; lorsqu'il s'identifie à des formes picturales ou musicales, au contraire, il tendrait vers son essence. À la différence du poète Blaise Cendrars, qui reproche au «rythme de L'Herbier» d'être "plutôt un rythme musical qu'un rythme proprement cinématographique», ajoutant : «Tout ce que les autres apportent au ciné, ce n'est plus du ciné» (p. 141), nombre de cinéastes et de théoriciens adhèrent à l'idée selon laquelle le modèle musical est susceptible d'aider le cinéma à viser son essence. Comment donc ce modèle agit-il?

Tout d'abord, il ne s'agit pas de se contenter d'adjoindre de la musique au cinéma. La mutité du cinéma n'est pas un défaut auquel il faudrait remédier par l'addition d'un art plus complet que lui. À l'instar de ce que pense Delacroix pour la peinture6, la notion de silence est, au contraire, marquée d'une connotation positive, la notion de mutité d'une valeur négative; le silence est l'absence de parole là où elle peut exister, la mutité son empêchement absolu. Le silence, c'est la maîtrise du bruit, la mutité, l'impuissance envers l'unique sonore. Au sujet du cinéma muet, divers auteurs s'avisèrent naturellement de cette distribution sémantique. Jean Tedesco remarque :

(...) on a dit: art muet. L'expression devrait être morte. Elle date de la découverte d'un art au fond du cinématographe; elle marque le sentiment de surprise répandu à l'époque, surprise de voir sur un écran des êtres s'agiter sans prononcer de paroles (p. 3).

«On a tort d'appeler le cinéma l'art muet, écrit Émile Vuillermoz; c'est en réalité l'art silencieux. Ne transformez pas en infirmité ce qui est une force cachée» (1927: p. 52). Nombreux sont ceux qui, comme Chaplin, mais pour des raisons différentes, pensent que «l'essence du cinéma, c'est le silence» (Lherminier, p. 252) 7.

Il faut donc préserver concrètement le «charme muet», en le considérant non comme un manque, mais comme une qualité, non comme une infirmité, mais comme une potentialité. Le silence fonde, d'abord, la capacité du film à se différencier de la réalité : une vague silencieuse, dit Kirsanoff qui parle du «silence du bruit», n'est plus la reproduction d'une vague réelle, mais un phénomène, 
une impression proprement cinématographique (1926: p. 9). On romprait le charme en ajoutant au film une musique dite additionnelle ou d'accompagnement, laquelle servirait, au mieux à étouffer le ronflement du projecteur ou à souligner le rythme de l'image, au pire, à pallier aux insuffisances de ce rythme; au contraire, le silence oblige le spectateur à se concentrer sur la forme visuelle 8 . Le rejet de la musique d'accompagnement ne signifie pas le rejet de la musique comme telle, mais le refus du collage sur un texte filmique travaillé avec précision d'une couche musicale en consonance approximative avec lui — du «papier peint sur des murs nus» selon la fameuse expression de Stravinsky. Certes, des partitions sont composées spécialement pour tel ou tel film et des compositeurs de talent apportent une contribution non négligeable (Busoni, Honneger, Satie, etc.). Il peut se faire qu'une composition ad hoc réalise un véritable montage audiovisuel plutôt que le collage incriminé. Toutefois, comme le remarque Louis Delluc : «La magnificience des partitions choisies est secondaire. Ce qu'il faut c'est que la musique s'adapte au film» (p. 10). À cette condition près, le cinéaste admet, un peu à contrecœur, la musique additionnelle; mais si d'autres la repoussent radicalement, ce n'est point qu'ils la méprisent. C'est plutôt qu'ils ont de la musique et du cinéma la même opinion, dans laquelle l'héritage conceptuel de la première pèse d'un grand poids. La musique additionnelle est généralement de faible qualité et, lorsqu'elle est de bonne qualité, elle n'est pas obligatoirement adéquate au film. Mais, par-delà le jugement esthétique, il y a l'essence même de la musique que les avant-gardistes conçoivent à la façon d'Apollinaire et de Kandinsky: un art pur par excellence, abstrait, mathématique, délié de toute adhérence naturaliste comme littéraire, dans lequel, comme dit Germaine Dulac, lorsqu'elle compare La Roue d'Abel Gance à un "poème symphonique», "le sentiment éclate non en faits et en actes, mais en sensation" (1927a : p. 48). On notera, au passage, que cette notion de poème symphonique ne semble pas pouvoir être confondue avec la forme musicale du même nom qui est justement une musique «à programme», c'est-à-dire dans laquelle la structure est fondée sur une trame anecdotique; toutefois, cette détermination narrative est aussi une libération formelle vis-à-vis des structures classiques, comme en témoignent La Symphonie fantastique de Berlioz ou La Mer de Debussy.

Dans La Roue, nous dit Germaine Dulac, l'image a «la valeur d'un son» (p. 48). Elle poursuit en étendant cette idée aux autres paramètres visuels : «Comme un musicien travaille le rythme et les sonorités d'une phrase musicale, le cinéaste se mit à travailler le rythme des images et leur sonorité» (p. 44). L'image comme son, la sonorité des images : faut-il entendre par là qu'il y a une 
musicalité de l'image, du cinéma, au sens où musicalité veut dire ce qui possède un caractère sensible de type musical, comme une voix musicale, une chaîne hi-fi musicale, une phrase ou un vers musicaux. Pour comprendre la chose, en effet, il convient de rappeler quelles sortes de correspondance Kandinsky, dans le cadre de l'analogie entre peinture et musique, prétend établir entre couleur et son :

Le rouge qu'on ne voit pas, mais que l'on conçoit de la manière la plus abstraite, éveille, néanmoins, une certaine représentation toute intérieure, à la fois précise et imprécise, d'une sonorité intérieure (p. 94).

La sonorité du cinéma n'est pas un son qui s'extérioriserait à partir de l'image, comme permettra de l'obtenir la sonorisation du film, mais la résonance que le visuel a dans notre esprit. Germaine Dulac généralise donc cette propriété à l'ensemble des paramètres du film :

Le mouvement cinégraphique, les rythmes visuels correspondant aux rythmes musicaux, qui donnent au mouvement général sa signification et sa force, faits de valeurs analogues aux valeurs harmoniques devaient se parfaire, si j'ose dire, des sonorités constituées par l'émotion contenue dans l'image elle-même (1927a : p. 44).

La musicalité a donc un autre sens, un sens plus intellectuel, plus abstrait : ce qui est propre, non à la matière, mais à la forme musicale, ce qui correspond donc à son caractère fondamentalement pur. La sonorité visuelle est la présence, en l'image, de modes formels qui s'apparentent à ceux de la musique. On ne les explore pas en adjoignant de la musique matérielle à un film quelconque, mais en explorant la musicalité même de l'image en tant que telle. Paul Ramain écrit :

(...) un beau film étant musical par lui-même, portant sa musique en lui, ne doit pas - sans hérésic - supporter un accompagnement musical adapté. Ainsi, en aucun moment la vision ne sacrifiera à l'audition, puisque audition et vision se trouvent incluses dans le film même» (1925b : p. 100).

Quant à la musicalité, "cet art doit se suffire à lui-même».

En quoi réside cette musicalité du cinéma? On peut répondre immédiatement : dans le mouvement. Étant donné que celui-ci est un trait distinctif du cinéma vis-à-vis de la peinture ou de la photographie, la musicalité du cinéma serait donc contenue dans son essence même comme médium. Mais le mouvement n'est-il pas aussi ce qu'exploite très exactement n'importe quel film qui reproduit la réalité vivante? 
Tout d'abord, le cinéma ne lut pour nous qu'un moyen photographique de reproduire le mouvement mécanique de la vie, le mot «mouvement» n'évoquant en notre esprit que la vision banale de gens et de choses animées, allant, venant, s'agitant, sans autre souci que d'évoluer dans le cadre d'un écran, alors qu'il eût fallu considérer le mouvement dans son essence mathématique et philosophique (Dulac 1927a: pp. 32-33).

L'agitation de la vie courante n'est pas mouvement (1924: p. 516); la simple monstration de la germination du blé manifeste mieux que la reproduction de cette agitation la conception «philosophique» du mouvement, en ce qu'elle introduit de l'ordre, une logique, dans le mouvement et, du même coup, produit «l'émotion purement visuelle» (1927b : p. 29). Or, cette logique est apportée par la distribution calculée de la durée que l'on nomme le rythme. Le poète Fernand Divoire affirme que «le cinéma, mouvement, a encore à se faire rythme» (1927: p. 43). Et c'est dans cette orientation que le cinéma rejoint la musique. «Tous les arts sont mouvement puisqu'il y a développement, dit Germaine Dulac, mais l'art des images est, je crois, plus proche par le rythme qui leur est commun» (1924 : p. 516). Ils sont légion les auteurs qui considèrent la notion de rythme comme dénominateur commun de la musique et du cinéma, et comme fondement de la spécificité de ce dernier. Le film dépasse le stade de la pure et simple reproduction de la réalité en imposant aux images elles-mêmes et à leurs combinaisons le rythme, c'est-à-dire une organisation particulière de la temporalité qui, pour certains auteurs inspirés par les théories des peintres, tel Canudo, se combine avec une organisation de la spatialité, le rythme plastique, dans une forme nouvelle englobant et dépassant la musique et la peinture (1911 : p. 169).

Cependant, il y a débat au sujet de la définition du rythme cinématographique. Pour Vuillermoz, il est la résultante de plusieurs mouvements - "c'est cette ordonnance et cette alternance des mouvements que l'on appelle généralement au cinéma, le rythme» (1927 : p. 46); pour Dulac, c'est le mouvement qui est la résultante de plusieurs rythmes - «(..) le rythme donne au mouvement sa signification intime» $(1927 b$ : p. 28). Or, cette divergence est significative. Ou bien on considère que la musicalité du film, procédera du travail que l'on imprime à l'image en la prenant pour telle; ou bien on considère qu'il faut traiter l'image musicalement, c'est-à-dire lui appliquer des procédés musicaux. «Nous voulons montrer aujourd'hui, écrit Paul Ramain, comment un film imaginé par un compositeur musicien peut devenir un vrai poème» $(1925 a:$ p. 100$)$ ou encore: il est 
(...) avantageux et indispensable pour l'avenir et l'autonomie du Cinéma de créer un film d'après une partition symphonique (...). Nous sommes persuadés que pour arriver au Cinéma intégral, il faut que le film passe par le stade de musique silencieuse (de Haas, p. $128)^{9}$.

De même Vuillermoz écrit «(...) la composition cinégraphique obéit, sans s'en douter, aux lois secrètes de la composition musicale. Un film s'écrit et s'orchestre comme une symphonie» (1919 : p. 3). Ce sont des musicologues qui parlent ainsi, employant un vocabulaire spécialisé plaqué sur les films (thèmedéveloppement, harmonie, mélodie, crescendo, orchestration, cadence, etc.) et préconisant la transposition au cinéma des modes de composition musicale. Vuillermoz va jusqu'à dire que les techniques des deux arts sont «rigoureusement semblables» (1927 : p. 59) et, lorsque Ramain en arrive au paradoxe de la «musique silencieuse», il nous faut régresser non loin de cette sorte de déficit qu'a longtemps sanctionné pour la peinture sa qualification de «poésie muette».

Pour l'autre tendance, si l'on peut dire, il ne s'agit pas d'aller chercher les outils dans la musique en tant que telle, mais tout simplement de développer le cinéma en tant que tel. À propos de La Roue, film-phare pour cette période, Germaine Dulac parle d'un «poème symphonique» ou d'une «symphonie visuelle», combinant les mêmes termes que Ramain ou Vuillermoz, mais elle ajoute entre parenthèses: «Le mot symphonie n'étant pris ici qu'en analogie» (1927a: p. 43). De même, Lionel Landry remarque, à juste titre, qu'«il n'y a probablement que très peu de rapports directs entre les rythmes musicaux et cinématiques» (p. 345). Différence dans la ressemblance ou ressemblance dans la différence, l'analogie permet, ici, de maintenir une distance entre les deux arts, en sorte que la musicalité de l'image n'a d'autres ressources que les paramètres spécifiques visuels et n'a d'autre effet que «l'émotion purement visuelle» selon l'expression de Germaine Dulac. Il ne s'agit plus, dans cette perspective d'importer les procédés rythmiques spécifiquement musicaux, mais d'utiliser ou de découvrir les procédés spécifiquement cinématographiques qui confèrent à l'image son «rythme intérieur» ou «extérieur» (Moussinac, p. 11) ${ }^{10}$, sa musicalité. Composition de l'image, procédés qui agissent sur le mouvement, montage : la musicalité du film serait l'exploitation des propriétés formelles du film. S'il y a analogie avec la musique, c'est du point de vue très général d'«une attache commune» entre elle et le cinéma, comme dit Dulac (1928 : p. 33), dans la mesure, en effet, où l'on peut s'élever des procédés musicaux et des procédés cinématographiques à quelques procédés 
encore plus formels qu'eux et qu'il n'est pas interdit, d'ailleurs, de désigner par des termes musicaux si ce degré de formalisme constitue un de leurs sens possible - par exemple, la cadence comme répétition de mouvements ou de sons qui se succèdent régulièrement: «Dans le cinéma, dit Dimitri Kirsanoff, il doit y avoir une cadence comparable à la cadence musicale. C'est ce rythme donné par le montage, qui doit créer la poésie de la vision» (1929 : p. 1). Au fond, les partisans supposés de l'importation, comme Vuillermoz, n'utilisent que des termes de cette sorte, et non pas, par exemple, la terminologie fine du solfège et de l'harmonie, en dépit du fait qu'ils se laissent aller à déclarer comme le musicologue : «Un film bien composé obéit instinctivement aux préceptes les plus classiques des traités de composition du Conservatoire» (1927 : p. 60). Non seulement il est douteux que ces principes appellent le seul instinct, mais encore la démonstration concrète de cette obéissance est fort improbable.

L'avant-garde des années 20 a puisé dans l'analogie avec la musique des forces pour construire un cinéma délié des adhérences littéraires, loin qu'il soit parvenu à en dépasser le caractère très général, loin que l'on ait produit des analyses précises de la musicalité du film. Les partisans de l'importation donnent l'impression de la précision en plaquant un embryon de vocabulaire technique tout aussi bien applicable à la littérature; les partisans de la spécificité, telle Dulac, offrent des descriptions de films convaincantes, sans élaborer le vocabulaire spécialisé qui eût rendu justice au cinéma de son aspiration à l'autonomie dans la pureté.

René Clair, qui demandait «une sensation de liberté musicale» en 1925 (de Haas, p. 128) ${ }^{11}$, préconisait déjà la même année de ruser avec les exigences commerciales, justifiant par avance son renoncement futur à assumer l'idéal de pureté (p. 271) ${ }^{12}$. La théorie de la musicalité du film, comprise comme accession du cinéma à l'art et comme conquête par ce médium de sa pureté, était destinée à disparaître dès les premiers balbutiements du parlant, du moins à titre provisoire. Pourtant, ses partisans n'étaient pas tout à fait démunis devant la sonorisation du film, du moins sur le plan théorique. Je terminerai cet article en évoquant rapidement ces deux «du moins»...

De même que la musicalité des images vise à les détacher de la réalité, il est possible de concevoir les sons autrement que comme une imitation de la nature, en référence toujours au modèle musical: Abel Gance parle d'«une musique de bruits pour ajouter à l'ambiance et non pas un calque servile des réalités qui nous entourent» (1929 : p. 10). Quant à Germaine Dulac, elle «soutient le cinéma sonore», comme prolongement dans la combinaison audiovisuelle des principes de la visualisation, à commencer par la 
musicalité: «Je sais qu'on peut jouer avec les bruits comme avec les images», dit-elle, et «j'emploie le film sonore à réaliser ma conception : faire une orchestration synchrone de bruits et d'images et non pas un enregistrement bâtard et banal de la musique» (1929: p. 12). Mais pour Henri Chomette, c'est le film sonore qui est «bâtard. En admettant même l'équilibre émotif de l'image sonore ou du visuel, on aboutit à un art hybride, nouveau sans être neuf, et qui sera aussi différent du cinéma même, que de la musique» (1929b: p. 66). Hormis les expériences abouties de Vertov (la composition «concrète» d'Enthousiasme, 1930), les intuitions expérimentales de Moholy-Nagy (écriture directe du son sur la pellicule) et, dans une moindre mesure, l'idée du contrepoint audiovisuel (manifeste d'Eisenstein-Poudovkine-Alexandrov), le cinéma sonore n'occupera jamais la place d'un art à part ni, $a$ fortiori, à part entière que lui prédisait Chomette, la bande-son devant être inéluctablement assujettie à la parole. Le verbe de la théorie ne pouvait pas résister à la fascination du verbe réel, de ce bavardage tant redouté par les partisans mêmes de l'image muette.

Mais ce n'est pas que ce projet, comme la plupart de ceux des cinéastes de l'avant-garde des années 20, était destiné à l'enterrement définitif. En tout cas, il refera surface avec l'avènement du cinéma dit underground, indépendant ou expérimental. Une étude récente d'Alain-Alcide Sudre sur Maya Deren indique combien les questions de la pureté et de la musicalité sont encore d'actualité à l'orée du renouveau de l'esprit d'avantgarde, même si elles n'affleurent que dans des périodes relativement limitées de l'histoire du cinéma. Ce cinéma en marge suit une progression qui n'est pas celle de l'industrie, justement parce qu'il prétend échapper aux contraintes de l'industrie. Chez Maya Deren, comme le montre Alain-Alcide Sudre, on retrouve la préoccupation de la visualité pure, de l'intégration des autres arts, avec son cortège d'exclusions (surtout la littérature et le théâtre) et de modèles autorisés (la musique et surtout la danse), du film silencieux et du cinéma sonore. Le cinéma dont elle fut l'inspiratrice développera les conséquences de ses expérimentations pratiques et théoriques, manifestant la résistance d'un projet dont il semblait, à tort, que l'avènement du parlant eût sanctionné la disparition irréversible. 
1 Pour réaliser ce travail, j'ai utilisé plusieurs ouvrages qui abordent le même sujet ou un sujet voisin. Notamment : Patrick de Haas, 1985, et Noureddine Ghali, 1989. La thèse de M. Ghali m'a considérablement facilité le travail, en raison de sa bibliographic monumentale, du nombre de citations de textes difficilement accessibles qu'clle contient et du large panorama des idées de l'avant-garde qu'elle développe — je le remercie vivement de sa collaboration involontaire...

2 «Un art doit apprendre d'un autre art l'emploi de ses moyens, même des plus particuliers et appliquer, ensuite, selon ses propres principes, les moyens qui sont à lui, et à lui seul. (...) Chaque art en s'approfondissant se renferme en soi et se sépare. Mais il se compare aux autres arts et l'identité de leurs tendances profondes le ramène à l'unité’ (Kandinsky, pp. 76-78).

3 Cinéa (13 mai 1921). Citć par Lherminier.

4 Autres propos d'Abel Gance : «Un grand fïlm? Musique : par le cristal des âmes qui se heurtent ou se cherchent, par l'harmonie des retours visuels, par la qualité même des silences. Peinture et sculpture par la composition; Architecture par la construction et l'ordonnancement; Poésie par les bouffées de rêve volées à l'âme des êtres et des choses; et Danse par le rythme intérieur qui se communique à l'âme et qui la fait sortir de vous et se mêler aux acteurs du drame.»

5 De même René Jeanne évoque un ceil qui «peut (...) voir ce qui n'est pas; tout ce qui appartient au domaine de l'imagination, de la fantaisie, du rêve (...)» (p. 27).

6 «Ce n'est pas un médiocre avantage pour la peinture, dit-il, que de n'être pas un art bavard", avantage qu'elle a sur les arts du temps (CEuvres littéraires, I, 94, cité par Olivier Revault d'Allonnes, p. 93). En effet, le bavardage, la prolixité, le délayage caractérisent aussi bien la musique (Journal, Paris, 11 mars 1849, éd. Joubin, p. 184) que la littérature (Ante, ler septembre 1859, pp. 744745). Maintes et maintes fois, Delacroix revient sur la supériorité du «charme muet» (Dieppe, 23 septembre 1854, p. 477) et, chaque fois, il l'attribue à la «puissance de la peinture» (Champrosay, 21 octobre 1853, p. 373). C'est ainsi qu'il «avoue sa prédilection pour les arts silencieux, pour ces choses muettes dont Poussin disait qu'il faisait profession» (Dieppe, 23 septembre 1854, p. 477). Le passage s'intitule «Sur le silence et les arts silencieux» et débute par un éloge du silence, de la discrétion, de la retenue. En filigrane, il y a cette critique du bavardage (ici, l'apanage des sots qui s'écoutent parler) dont nous avons vu quel parti le peintre sut tirer.

7 Motion Picture Magazine (1930). Cité par Lherminier. Chaplin propose cette formule dans le cadre d'une défense et illustration du cinéma muet comme art de la pantomime.

8 La musique «distrait le spectateur et ne sert qu'à masquer en général la pauvreté du rythme cinégraphique, la lenteur de l'action et la laideur du décor. (...) Le silence nous obligera par l'éducation de notre sens visuel, à apprendre à regarder, à acquérir une vision plus pénétrante, plus lyrique, plus réelle» : texte anonyme paru dans le Bulletin du Club du Cinéma, Ostende (17 octobre 1928). Cité par de Haas, p. 127.

9 Paul Ramain, 1925b, citć par de Haas.

10 Le rythme intérieur concerne la «représentation (décors, costumes, éclairages, objectifs, angles de prise de vue) et l'interprétation», le rythme extérieur, «le découpage, (...) la valeur en durée, mathématique ou sentimentale, donnée à chaque image, par rapport aux images qui la précèdent et à celles qui la 
suivent». Cette «distinction organique», dit l'auteur, caractérise les deux composantes du «rythme général du film».

11 René Clair, 1925a. Cité par de Haas.

12 René Clair, 1925b. Cité par de Haas.

\section{BIBLIOGRAPHIE}

Apollinaire, Guillaume. Méditations esthétiques. Les peintres cubistes. Paris : éd. Breunig-Chevalier, 1980.

Canudo, Ricciotto. «La Naissance d'un sixième Art - Essai sur le Cinématographe». Les Entretiens Idéalistes no LXI, 6e année, tome X (25 octobre 1911).

Canudo, Ricciotto. Cinéa (1.3 mai 1921). Cité par Lherminier, p. 29.

Canudo, Ricciotto. «L'Esthétique du Septième art. - II. Le Drame visuel». Le Film no 181, 8e annéc (mai-juin 1921).

Canudo, Ricciotto. «Manifeste des Sept Arts». Gazette des Sept Arts no 2 (25 janvier 1923) p. 2.

Canudo, Ricciotto. "Chronique du Septième Art - Films sans images». Parismidi no 4.194, 13e annéc (2 novembre 1923) p. 1.

Cendrars, Blaise. «Entretien avec François et André Berge». Les Cahiers du Mois no 16-17 (octobre 1925).

Chomette, Henri. «Interview de Maurice Maigance». L'Ami du peuple (28 janvier 1929).

Chomette, Henri. «Innovations industrielles». Cinéma no 18 (janvier 1929).

Clair, René. «Rythme». Les Cahiers du Mois nos 16-17 (octobre 1925).

Clair, René. «Cinéma pur et cinéma commercial». Les Cahiers du mois no 16-17 (octobre 1925).

De Haas, Patrick. Cinéma intégral. De la peinture au cinéma dans les années vingt. Paris : Transédition, 1985.

Delacroix, Eugène. Journal, 1822-1863. Paris : Plon, 1981.

Delluc, Louis. «Le commerce du cinéma». Cinéa no 24 (21 octobre 1921).

Divoire, Fernand. «Le commerce du cinéma». Cinéa no 24 (21 octobre 1921).

Divoire, Fernand. «Danse et cinćma». Schémas no 1 (février 1927).

Dulac, Germaine. «Conférence à la Société des Amis du Cinéma, 7 décembre». Cinémagazine no 51 (19 décembre 1924).

Dulac, Germaine. "L'essence du cinéma - l'idée visuelle». Les Cahiers du mois nos 16-17 (1925).

Dulac, Germaine. «Les esthétiques, les entraves, la cinégraphie intégrale». L'Art cinématographique, tome II. Paris : Librairie Félix Alcan, 1927.

Dulac, Germaine. «Du sentiment à la ligne». Schémas no 1 (février 1927).

Dulac, Germaine. «Films visucls et anti-visuels». Le Rouge et le Noir (juillet 1928).

Dulac, Germaine. «Réponse à l'enquête "Le film sonore - Qu'en pensent nos réalisateurs?"». Cinéa-Ciné pour tous no 138 (1er et 15 août 1929).

Epstein, Jean. Bonjour cinéma. Paris : Éditions de la Sirène, 1921. 
Fescourt, Henri et Jean-Louis Bouquet. L'Idée et l'Écran. Opinions sur le cinéma, fascicule I. Paris : Imprimerie G. Haberschill et A. Sergent, 1925.

Gance, Abel. «Le temps de l'image est venu». L'Art cinématographique, tome II. Paris : Librairie Félix Alcan, 1927.

Gance, Abel. «Réponse à l'enquête "Le lïlm sonore - Qu'en pensent nos réalisateurs?"». Cinéa-Ciné pour tous no) 138 (ler et 15 août 1929).

Ghali, Noureddine. L'"avant-garde" cinématographique en France durant les années vingt : idées, conceptions, théories. Thèse présentée à l'Université de Paris I, 1989.

Gorelof. "Suggérer...». Cinéa-Ciné pour tous no 91 (15 août 1927).

Jeanne, René. «La controverse de la couleur». Cinéa-Ciné pour tous no 78 (1er février 1927).

Kandinsky. Du spirituel dans l'arl, 1911. Paris : Denoël/Gonthier, 1969.

Kirsanoff, Dimitri. «Les problèmes de la photogénie». Cinéa-Ciné pour tous no 62 (1er juin 1926).

Kirsanoff, Dimitri. «Entretien avec Marcel Lapierre». Bordeaux-Ciné no 18 (25 janvier 1929).

Landry, Lionel. «Le rythme». Cinémagazine no 21 (24 mai 1929).

Lherminier, Pierre. L'Art du cinéma. Paris : Seghers, 1960.

Mallarmé, Stéphane. «La Musique et les Lettres», conférence 1895. Igitur. Divagations. Un coup de dés. Paris : Poésie/Galllimard, NRF, 1976.

Moussinac, Léon. «Le cinéma au Salon d'automne». Gazette des Sept Arts no 9 (1er novembre 1923).

Pierre-Quint, Léon. «Signilication du cinéma». L'Art cinématographique, tome II. Paris : Librairie Félix Alcan, 1927.

Picabia, Francis. «Instantanéismc». Comoedia (21 novembre 1924).

Ramain, Paul. «Musique, Art et Cinéma». Le Courrier musical et théâtral no 3 et no 4 (1er et 15 février 1925).

Ramain, Paul. «L'influence du cinéma sur la musique». Les Cahiers du Mois $\mathrm{n}^{\mathrm{OS}} 16-17$ (1925).

Ramain, Paul. «Sensibilité intelligente d'abord, objectif ensuite». Cinéa-ciné pour tous no 55 (15 févricr 1926).

Revault D'Allonnes, Olivier. La Création artistique et les Promesses de la liberté. Paris : Klincksieck, 1973.

Sudre, Alain-Alcide. Dialogues théoriques avec Maya Deren. Du film expérimental à l'expérience ethnographique. Thèse d'État présentée à l'Université de Paris X, 1991.

Tedesco, Jean. «Opinions-Art silencieux». Comøedia, no 4372 (11 décembre 1924).

Vuillermoz, Émile. «Chroniquc - Devant l'Écran». Le Temps no 20.381 (25 avril 1917).

Vuillermoz, Émile. «Devant l'écran - Lueurs». Le Temps no 21.150 (4 juin 1919).

Vuillermoz, Émile. «La musique des images». L'Art cinématographique, tome III. Paris : Librairie Félix Alcan, 1927. 